\title{
VIOLENCIA ÍNTIMA EN PAREJAS JÓVENES DEL MISMO SEXO EN CHILE
}

\author{
Claudia SALDivia Mansilla ${ }^{1}$ \\ BÁRBARA FAÚNDEZ REYES ${ }^{2}$ \\ SEBASTIÁN SOTOMAYOR LLANOS ${ }^{3}$ \\ FREDY CEA LEIVA ${ }^{4}$
}

\begin{abstract}
RESUMEN:
Esta investigación tuvo por objetivo caracterizar la violencia intima de parejas jóvenes del mismo sexo en Chile. La muestra estuvo compuesta por 631 jóvenes, de 18 a 29 años de edad, quienes respondieron un cuestionario sobre violencia de pareja, mediante una plataforma de internet que fue difundida por redes sociales. Los resultados obtenidos señalan que el $84 \%$ de los jóvenes de esta muestra viven violencia de pareja. Un $80,5 \%$ vive violencia psicológica, un 31,2\% violencia física, y un 48,8\% violencia sexual. Existe relación entre algunos tipos de violencia y variables género y nivel educacional. Los resultados son discutidos en base a hallazgos de investigaciones internacionales en el tema.
\end{abstract}

PALABRAS CLAVE: VIOLENCIA DE PAREJA, JÓVENES, HOMOSEXUALIDAD

${ }^{1}$ Chilena, Psicóloga, Magister en Psicología, académico Universidad Santo Tomás, claudiasaldivia@santotomas.cl

${ }^{2}$ Chilena, Psicóloga, Licenciada en Psicología, barbarafaundezreyes@gmail.com

${ }^{3}$ Chileno, Psicólogo, Licenciado en Psicología, sebastiansotomayor@outlook.com

${ }^{4}$ Chileno, Sociólogo, Magister en Gerencia Social, académico Universidad Santo Tomás, fredycea@santotomas.cl

Los autores agradecemos el apoyo de Fundación Iguales-Chile para el desarrollo de esta investigación en cuanto a su difusión en redes sociales y divulgación de resultados. 


\title{
VIOLÊNCIA ÍNTIMA EM CASAIS JOVENS DO MESMO SEXO NO CHILE
}

\begin{abstract}
RESUMO
Esta pesquisa teve por objetivo caracterizar a violência íntima nos casais jovens do mesmo sexo no Chile. A amostra esteve composta de 631 jovens, entre 18 e 29 anos de idade, que responderam a um questionário sobre a violência no relacionamento, através de uma plataforma na internet espalhada pelas redes sociais. Os resultados obtidos indicam que $84 \%$ dos jovens desta amostra vivem violência no relacionamento. Sendo 80,5\% violência psicológica, 31,2\% violência física e 48,8\% violência sexual. Existe relação entre alguns tipos de violência e variáveis gênero e nível de escolaridade. Os resultados são discutidos baseados nos resultados de pesquisas internacionais sobre o assunto.
\end{abstract}

PALAVRAS-CHAVE: VIOLÊNCIA NO RELACIONAMENTO - JOVENS -

HOMOSSEXUALIDADE.

\section{INTIMATE PARTNER VIOLENCE IN HOMOSEXUAL YOUNG COUPLES IN CHILE}

\begin{abstract}
This research aims to characterize intimate partner violence in homosexual Chilean young couples. From a sample of 631 people aged 18 to 29 who answered questions about intimate partner violence through an online questionnaire spread across social networks, the results obtained show an $84 \%$ of young people who are victims of intimate partner violence, $80,5 \%$ of psychological violence while $31,2 \%$ suffer from physical violence and $48,8 \%$ from sexual violence. There is a relationship between some types of violence and the variables of gender and education level. The results are discussed based on findings of international topic-related studies.
\end{abstract}

KEY WORDS: INTIMATE PARTNER VIOLENCE - YOUNG PEOPLE - HOMOSEXUALITY. 


\section{INTRODUCCIÓN}

En esta investigación se aborda la problemática de la violencia íntima de parejas jóvenes del mismo sexo en Chile, como un problema social importante, pero a la vez invisibilizado, debido a que la temática de las minorías sexuales ha sido poco estudiada a nivel académico en nuestro país. Según investigadores del tema, los estudios en homosexuales y lesbianas relacionados al maltrato son un fenómeno poco estudiado, en cambio la tasa de publicación que examina la dinámica de la violencia heterosexual ha superado con creces este tipo de investigaciones (Murray, Mobley, Buford \& Seaman-Dejohn, 2007; Ortega, 2014). En relación a la violencia de pareja en jóvenes, ocurre un fenómeno similar, dado que existen investigaciones a nivel internacional y algunas a nivel nacional respecto al tema en jóvenes heterosexuales (Vizcarra y Poo, 2011; Vivanco, Espinoza, Romo, Véliz y Vargas, 2015), pero es menor la investigación en parejas de jóvenes homosexuales (Finneran \& Stephenson, 2014). De hecho, en Chile es prácticamente inexistente.

Históricamente, la violencia íntima entre parejas del mismo sexo ha sido pasada por alto por la comunidad homosexual debido a asuntos que se consideran más importantes o urgentes en esta comunidad. Ejemplo de esto son la homofobia y el heterosexismo que se han abordado dentro de los ámbitos político y jurídico, en los argumentos a favor de los matrimonios del mismo sexo y beneficios a las parejas. También las cuestiones relativas al VIH / SIDA y la discriminación en la escuela y en el trabajo han recibido mucha atención (Ortega, 2014).

Sin embargo, el maltrato en parejas homosexuales ha sido identificado como un problema preocupante en la comunidad homosexual, de hecho, ha sido nombrado el tercer mayor problema después del VIH y el consumo de sustancias (Ortega, 2014). Más aún, se identifica a los jóvenes de estas minorías como quienes sufren con mayor frecuencia violencia de pareja (Informe National Coalition of Anti-violence Programs (NCAVP), 2014). Esto no deja 
de ser un asunto preocupante, considerando que sería en la etapa de juventud donde se consolidan ciertos patrones de relación de pareja que pueden desembocar en una cronificación de la violencia de pareja y/o en otros problemas de salud y sociales en etapa adulta (Cortés-Ayala, Flores, Bringas, Rodríguez-Franco, López-Cepero y Rodríguez, 2015:6).

\section{ASPECTOS GENERALES DE LA VIOLENCIA DE PAREJA EN JÓVENES.}

El fenómeno de la violencia de pareja en jóvenes se constituye en un problema social y de salud pública importante, dado que los estudios revelan una alta prevalencia de este tipo de violencia en jóvenes de distintos países. Los niveles trascienden la clase social, el origen étnico, el nivel socio-económico, el nivel educativo o la orientación sexual (López y Ayala, 2011).

La violencia de pareja en jóvenes ha sido definida por algunos autores como el "ejercicio o amenaza de un acto de violencia por al menos un miembro de una pareja no casada sobre el otro, dentro del contexto de una relación romántica” (Sugarman \& Hotaling, en Poo y Vizcarra, 2008: 82).

Ortega (2014) encuentra diferencias entre violencia y abuso afirmando que violencia es un comportamiento, una acción, mientras que abuso es un patrón de intimidación y control, que incluye la violencia dentro de un contexto de poder y dinámicas de control en una relación íntima, y que causa daño psicológico y a menudo físico. El abuso físico es la imposición intencional o intentar causar daño corporal a otra persona, se produce en un contexto de dinámicas de poder y de control con la pareja abusiva, aspirando a lograr y mantener el control sobre su pareja a través del uso de la fuerza física (Murray \& Mobley, 2009:362), mientras que la violencia física, según Alberdi y Matas (2002), corresponde a una escalada que es progresiva y que puede comenzar con un empujón o pellizco y llegar a lesiones graves y en casos extremos al homicidio. 
En tanto, la violencia psicológica se asocia a la desvalorización de la otra persona (Alberdi y Matas, 2002). Algunos de los comportamientos a través de los cuales se manifiesta son la ridiculización, amenazas verbales e insultos, humillación, acciones que afectan la autoestima y el bienestar psicológico de la persona (Vivanco et al., 2015). En tanto Murray \& Mobley (2009) definen el abuso psicológico y emocional como intentos de una persona abusiva para interrumpir el bienestar mental y/o afectivo de su pareja.

La violencia sexual se da "mediante presiones físicas o psíquicas que imponen relaciones o actos sexuales no deseados mediante coacción, intimidación o indefensión” (Alberdi y Matas, 2002:93), en cuanto al abuso sexual se incluyen actos forzados de naturaleza sexual que los perpetradores utilizan para asegurar o mantener su supremacía sobre sus parejas (Murray et al., 2007:16).

En Chile, durante el año 2013 el Instituto Nacional de la Juventud (INJUV, 2013) realizó la Séptima Encuesta Nacional de la Juventud, donde señala que los jóvenes chilenos declaran en un 16\% haber experimentado violencia en su relación de pareja, pero este porcentaje aumenta a 30\% cuando se consulta por “conductas de control”, que los jóvenes no asumen como conductas violentas, pero que serían una forma de violencia psicológica (ReyAnacona, 2013). En una investigación reciente realizada con jóvenes que cursan educación superior en la ciudad de Osorno, Vivanco et al. (2015) encontraron una prevalencia de 85,3\% de violencia recibida al menos en una ocasión y un 85,6\% de violencia ejercida en al menos una ocasión, considerando distintos tipos de violencia: psicológica, emocional, física, económica y sexual.

Estos tipos de violencia surgen de ciertos factores que pueden influir y/o precipitar su aparición, y que son de tipo individual, relacional y social. Algunos de estos factores son: la observación de violencia entre los padres, la aceptación de la violencia en la relación de pareja, tener amigos o conocidos que han sido víctimas o victimarios de dicha violencia, los roles tradicionales 
de género y la experiencia de haber sido víctima de violencia por parte de la familia de origen (Rey -Anacona, 2008; Sanhueza, 2016).

Los principales factores de riesgo se relacionan mayoritariamente con factores de interacción social, sea desde la temprana infancia en la relación con los propios cuidadores, hasta futuras relaciones maduras, además de la propia cultura patriarcal que implanta la sociedad actual (Rey-Anacona, 2008; Sanhueza, 2016). Otros factores sociales son la aceptación y justificación de la violencia que incrementa la probabilidad de ejecutar actos de maltrato hacia la pareja, tanto si la víctima como el/la victimario/a aceptan su ejercicio como algo natural o posible en la relación de pareja (Rey-Anacona, 2008).

A pesar de que puedan existir algunas similitudes entre violencia de pareja heterosexual y homosexual, se afirma que los paradigmas heterosexuales de la violencia de pareja, aunque valiosos, no pueden explicar plenamente la experiencia de la violencia íntima en parejas del mismo sexo (Murray et al., 2007; Ortega, 2014; Brown \& Herman, 2015).

\section{VIOLENCIA EN PAREJAS DEL MISMO SEXO}

Para comprender mejor esta temática, se definirá identidad sexual como la "sensación interna de identificación o falta de identificación que una persona tiene en relación a su sexo biológico” (Mujika y Ureta, 2007:4) y homosexualidad como un término que ayuda a referirse a la inclinación del deseo del sujeto hacia una persona de su mismo sexo. Lozano (2009) hace una distinción entre la homosexualidad y lo gay, entendiendo éste último como "un conjunto de vivencias sociales que estructuran una subjetividad específica, con sus propios ritos de paso, sus mitos fundantes, sus territorios de reconocimiento y sus usos especiales del lenguaje” (156). Además se puede definir las parejas del mismo sexo como dos personas homosexuales, o de orientación sexual bisexual, que actualmente comparten una importante relación interpersonal 
afectiva, típicamente caracterizados por las conexiones románticas, sexuales, emocionales, y otros (Murray et al., 2007).

La orientación sexual, según la Agencia De Derechos Fundamentales De La Unión Europea (FRA, 2009) se define como "la capacidad individual de sentir una profunda atracción emocional, afectiva y sexual por personas de un género diferente, del mismo género o de más de un género, así como de mantener relaciones íntimas y sexuales con ellas” (7).

La definición de género, por su parte, ha sido contextualizada como:

Conjunto de creencias, prescripciones y atribuciones que se construyen socialmente tomando a la diferencia sexual como base. Esta construcción social funciona como una especie de «filtro» cultural con el cual se interpreta al mundo, y también como una especie de armadura con la que se constriñen las decisiones y oportunidades de las personas dependiendo de si tienen cuerpo de mujer o cuerpo de hombre. Todas las sociedades clasifican qué es «lo propio» de las mujeres y «lo propio» de los hombres, y desde esas ideas culturales se establecen las obligaciones sociales de cada sexo, con una serie de prohibiciones simbólicas. (Lamas, citada en Amar, 2012:3)

Cantera (2004) propone aplicar un modelo explicativo psicosocial independiente del género, que contemple la diversidad sociocultural de los procesos de violencia en la pareja. Sostiene que las teorías feministas acerca de la violencia doméstica son heterocentristas y víctimacentristas y que impiden visualizar y nombrar la violencia en parejas homosexuales.

Es por esto que resulta importante identificar y describir los tipos de violencia dentro de las parejas homosexuales, ya que al no tener conocimientos sobre esta realidad se vuelve más vulnerable a esta minoría (Cantera, 2004). Además, dentro de este grupo, serían los jóvenes los más vulnerables, tanto por el alto porcentaje de violencia de pareja encontrado (Informe NCAVP, 2014), 
como por la etapa de vida que atraviesan, siendo también más vulnerables a sufrir abusos y conductas de violencia por parte de sus pares y su familia (Stephenson, Hast, Finneran \& Sineath, 2014; Pérez, Ramos y de la Vega, 2016).

Debido a lo mencionado anteriormente, se puede decir que las secuelas de la violencia de pareja del mismo sexo son significativamente diferentes a las producidas por la violencia en parejas heterosexuales $y$, por otra parte, el impacto de este tipo de violencia en la etapa juvenil es considerable.

Es así como las personas homosexuales tienen menos probabilidades de obtener el apoyo social y de organización para la violencia de pareja en sistemas judiciales o servicios de salud mental de la comunidad (Edwards \& Sylaska, 2013; Sorenson \& Thomas, 2009; Brown \& Herman, 2015).

Villalón (2015) concluye que la invisibilización de la violencia en las parejas del mismo sexo, es un tipo de violencia que se da dentro de parejas que ya viven su sexualidad de una forma mucho menos visible que las heterosexuales, puesto que no se adecuan a la heteronormatividad impuesta. El hecho de reconocer la violencia en las parejas del mismo sexo es más complicado, ya que es difícil reconocerse como víctima en un ambiente de invisibilidad. A diferencia de la violencia de género, que hace años se trabaja para su visibilización.

En el caso de los jóvenes homosexuales, esta visibilización se complica aún más, ya que están enfrentando el proceso de consolidación de su identidad sexual, que muchas veces conlleva discriminación por parte del entorno cercano, por lo que existe un auto-rechazo que lleva a no reconocer el hecho de tener una pareja del mismo sexo (Fernández y Vásquez, 2013; Barrientos, Gutiérrez, Ramírez, Vega y Zaffirri, 2016).

El rol del género, específicamente un alto nivel de masculinidad, es un predictor sobresaliente de la violencia de pareja, tanto en adultos como en 
jóvenes. Se encontró que cuanto más un hombre o una mujer homosexual se identifica con los componentes de la personalidad masculina, es más probable que él o ella pudiesen convertirse en abusivos (McKenry, Serovich, Mason y Mosack, 2006; Tellez y Walters, 2011; Stephenson et al., 2014).

La precariedad económica también podría explicar el conflicto y el abuso en las relaciones homosexuales y lésbicas, especialmente cuando lleva a sentimientos de inferioridad. Estos sentimientos también pueden ser provocados por el tamaño físico, el atractivo y la estabilidad del trabajo, generándose así una inconsistencia de estatus en las relaciones homosexuales y lésbicas que pueden contribuir a la violencia (McKenry et al., 2006:238). Si bien esto se ha descrito en parejas homosexuales adultas, es probable que también ocurra en la relaciones de pareja entre jóvenes, especialmente por el atractivo físico y las diferencias socioeconómicas (Stephenson et al., 2014).

Otro factor descrito asociado a la violencia íntima de pareja en jóvenes del mismo sexo, es la obtención de control sobre el otro miembro de la pareja, para elevar de forma explícita el poder de una persona sobre otra. La dependencia económica y emocional son factores tanto en el riesgo de victimización como en el riesgo de perpetrar la violencia íntima de pareja (Téllez \& Walters, 2011). La violencia física puede ser utilizada por los perpetradores, ya que es un medio muy eficaz para obtener el control sobre otra persona pero las diversas formas de abuso psicológico pueden ser más comunes para sostener el abuso dentro de la misma (Ibíd.).

Ortega (2014) propone componentes de abuso específicos de parejas homosexuales, adultos y jóvenes. Entre ellos, el abuso sobre la identidad sexual, donde el maltratador utilizaría su conocimiento sobre los estereotipos que definen como son los homosexuales en general para abusar de su pareja, por no mantener relaciones sexuales anales. Así también, la "salida forzosa" (“outing forzoso"), que consiste en revelar la orientación sexual de una persona 
sin su consentimiento o amenazarle con hacerlo en su trabajo, ante su familia, amigos o agencias de adopción (Ibíd.; Tellez \&Walters, 2011).

\section{Estudios}

En Estados Unidos se realizó una investigación con una muestra representativa nacional de estudiantes secundarios, en que el 29,7\% de la muestra reportó haber sufrido algún tipo de violencia de pareja en relaciones del mismo sexo; el 19,9\% indicó que había experimentado violencia, el 12,5\% informó violencia psicológica, y el 10,5\% violencia sexual (Edwards \& Sylaska, 2013). Estos mismos autores citan un estudio del 2012 que encontró una prevalencia de $43.5 \%$ de violencia de pareja en estudiantes secundarios LGBT (Jones \& Raghavan, en Edwards \& Sylaska, 2013).

Así también, una revisión de investigaciones en el tema realizada por Brown \& Herman (2015) encontró una estimación entre un 8,8\% a 56,9\% de violencia intima de pareja en jóvenes LGTB (lesbianas-gays-transexualesbisexuales), en distintos estudios realizados la última década en Estados Unidos.

Un estudio español realizado el año 2014 con lesbianas jóvenes y adultas, indica una prevalencia de $48 \%$ de violencia de pareja a lo largo de la vida, y un 43\% de perpetración (Badenes et al., 2014, citados en Villalón, 2015). En esta investigación, además, es la violencia psicológica la que prevalece en las parejas de mujeres auto identificadas como lesbianas, principalmente las humillaciones, las críticas no constructivas, los gritos, las amenazas, etc. (Ibíd.).

En una investigación realizada a parejas de hombres jóvenes y adultos, homosexuales y bisexuales en Argentina y España, efectuada por Ortega (2014), con respecto a la victimización, obtuvo que el 70\% de la muestra residente en España y un 76,87\% de la muestra residente en Argentina se 
consideran víctimas de agresión psicológica y un 26,78\% en España por un 35,87\% en Argentina víctimas de agresión física, encontrando también una prevalencia significativa en victimización sexual con un 43,2\% y un 51,23\% en España y Argentina, respectivamente, considerándose victimas de agresión sexual. Además, da cuenta de que, en relación a la edad, tanto en la muestra argentina como en la española las mayores tasas de agresión se producen entre los 18 años a 29 años, disminuyendo en los siguientes grupos de edad (Ibíd.).

Como refiere Cantera (2004), “todos los intentos de explicar la violencia en pareja homosexual parten del reconocimiento implícito de la existencia de la misma. Algunos estudios empíricos tratan simplemente de describir y de documentarlo, lo cual resulta absolutamente imprescindible para progresar en el conocimiento de la misma” (129).

Según los resultados del Instituto Nacional de Estadísticas (INE, 2012), $34.976(0,36 \%)$ personas declaran convivir con parejas del mismo sexo. Según la Séptima Encuesta Nacional de la Juventud (2013), el 2\% del total de la población juvenil se autodefine homosexual y el 10\% declara no ser heterosexual. Por su parte, el XIII Informe anual de derechos humanos de la diversidad sexual en Chile, efectuado el año 2014 por el Movimiento de integración y liberación homosexual (MOVILH, 2015), señala que el 69\% de los jóvenes declara tener amigos o amigas LGBT, el 24\% reconoce que en su familia hay personas de la diversidad sexual y el 15\% ha participado en manifestaciones a favor de las minorías sexuales.

No obstante lo anterior, que sugiere un aumento y reconocimiento explícito en los jóvenes en la existencia de relaciones de pareja no heterosexuales, la opinión pública en Chile sobre este tema es algo diferente. Un estudio realizado por el INJUV y citado en el informe referenciado anteriormente, con jóvenes de 15 a 29 años, señala que el 31\% cree que los derechos de la diversidad sexual han avanzado "bastante o mucho" en los 
últimos 10 años, el 25\% piensa que “algo" y el 42\% considera que "poco o nada”.

Las políticas sociales en Chile han abordado el fenómeno de la violencia en parejas jóvenes durante los últimos cinco años, sin embargo, no han abordado este fenómeno en los jóvenes homosexuales. Esta investigación pretende aportar al conocimiento de este fenómeno para visibilizar en la opinión pública esta problemática soterrada, y contribuir a promover políticas públicas de prevención e intervención en estas parejas. Es relevante obtener este tipo de información de investigaciones científicas, y así contar con una base objetiva para poder intervenir en el fenómeno.

A partir de lo anterior, el objetivo de la presente investigación fue caracterizar el tipo de violencia en parejas jóvenes del mismo sexo en Chile durante el año 2016. Para esto se describe la violencia psicológica, violencia física y violencia sexual. Además se buscó determinar la relación existente entre variables socio-demográficas (género, edad, nivel educacional) y la violencia de pareja, en los jóvenes del estudio.

\section{Metodología}

El diseño utilizado en esta investigación fue un diseño transversal correlacional, dado que se efectuó la medición de variables en la muestra definida en un solo momento, y se buscó, de acuerdo a los objetivos planteados, caracterizar el fenómeno de la violencia de pareja en jóvenes homosexuales, no solo a través de su descripción, sino de la posible correlación con algunas variables socio-demográficas como género, edad y nivel educacional (Hernández, Fernández y Baptista, 2010).

La población estimada para llevar a cabo esta investigación se obtuvo mediante un cruce de información entre datos recogidos del último censo (2012) realizado por el Instituto Nacional de Estadísticas (INE, 2012), el cual 
indica que 2.383.196 personas son jóvenes entre 18 a 29 años de edad, y la Séptima Encuesta Nacional de la Juventud (INJUV, 2013) donde el 2\% de los jóvenes declara ser homosexual, entregando una población estimada de 47.664 jóvenes homosexuales en Chile. La muestra obtenida fue de 631 personas con un intervalo de confianza del 95\%, y un margen de error de $3.8 \%$.

Los criterios de inclusión de la muestra fueron:

- Hombres y mujeres de orientación sexual homosexual.

- Nacionalidad Chilena.

- Edades entre 18 a 29 años.

- Estar o haber estado en una relación de pareja de al menos 6 meses.

Como criterio de exclusión, se señaló que los participantes no hayan estado nunca en una relación de pareja de al menos 6 meses. Esto debido a que algunas investigaciones en la temática reportan que la extensión temporal de la relación de pareja, aumenta la probabilidad de que se perciban conductas de violencia, estableciendo un tiempo de relación mínimo de tres a seis meses (Tucker, Young, Waller, Martin \& Kupper, 2004; González, 2008).

La muestra fue no probabilística, porque estuvo dirigida específicamente a personas jóvenes que sean homosexuales y accedan a redes sociales, y desde ellos poder caracterizar la violencia de pareja. Estuvo constituida por 631 personas que mantuvieron una relación de pareja del mismo sexo durante un periodo de al menos 6 meses.

El 64,8\% de los participantes se constituyó por mujeres, mientras que el $35,2 \%$ por hombres. El 39,3 \% se encuentra entre los 18 a 23 años y 60,7 \% se encuentra entre los 24 a 29 años de edad. En cuanto al nivel educacional, el 10,3\% cuenta con educación media completa, un $43,7 \%$ de los participantes no ha completado la educación superior, mientras que el 37,9\% cuenta con educación superior completa y el 8,1\% cuenta con educación de postgrado. En 
relación a la caracterización demográfica, el 48\% pertenece a la región Metropolitana, mientras que el 9,8\% pertenece a la zona norte del país, el $21,3 \%$ a la zona centro y el 20,9\% a la zona sur.

\section{INSTRUMENTO.}

El cuestionario utilizado fue construido por Vizcarra \& Poo (2011), tomando como referencia la literatura nacional e internacional respecto al tema. Éste fue sometido al arbitrio de tres jueces expertos, quienes evaluaron la validez de constructo del instrumento. El instrumento se creó para evaluar la violencia de pareja en jóvenes y está compuesto por tres dimensiones: violencia psicológica, violencia física y violencia sexual. La subescala violencia psicológica utiliza 11 ítems, con un Alfa de Cronbach de 0,79; violencia física utiliza 6 ítems, con un Alfa de Cronbach de 0,69 y violencia sexual está compuesta de 7 ítems con un Alfa de Cronbach de 0,64. Todas las subescalas tienen el mismo formato, con cuatro opciones de respuesta, (nunca; 1 o 2 veces; 3 a 5 veces; 5 o más veces) con valores de 0 (nunca) a 3 (5 o más veces).

Para el uso en esta investigación, se revisó cada uno de los ítems que componen el instrumento donde, como resultado final, en el ítem 4 se cambia la redacción para adaptar a la población dirigida, y el ítem 14 se omite, ya que no corresponde a las características de la población medida. El nivel de confiabilidad del instrumento en esta investigación, determinado a través del estadístico Alfa de Cronbach, fue de 0,75. Para la escala violencia psicológica fue de 0,65; para la escala de violencia física fue 0,71 y para la escala de violencia sexual fue de 0,76 .

\section{Procedimiento.}

Esta investigación fue presentada y aprobada en el Comité de Ética Científico de la Universidad Santo Tomás. Las personas participantes fueron 
contactadas mediante redes sociales tales como Twitter, Whatsapp, Facebook e Instagram, donde se adjuntó el enlace de la encuesta ya estructurada, de manera que las personas que leyeron y aceptaron el consentimiento informado, que señala que la participación es voluntaria, anónima y confidencial, pudieron acceder a contestar el cuestionario en línea. También se les consultó acerca de variables socio demográficas como género, edad, nivel educacional y región de procedencia. La difusión de dicha encuesta fue realizada por las redes de los investigadores, destacando Fundación Iguales Chile, institución que se dedica a trabajar en el tema de diversidad sexual.

Como resguardo ético, se señaló en el consentimiento informado que, en caso de sentirse afectados por el contenido de las preguntas, se podrían contactar con la investigadora principal, quien realizaría el contacto con el Centro de Atención Psicológica de la Universidad Santo Tomás (existente en diferentes ciudades del país), para prestar ayuda psicológica en la sede de la universidad más cercana a la persona a través de la atención directa o la derivación pertinente. La encuesta tuvo una duración de 20 minutos aproximadamente.

\section{Resultados}

Para el análisis de los datos se utilizó el programa estadístico SPSS versión 19. Se utilizaron tablas de frecuencia y estadísticos descriptivos como media y desviación estándar. Para asociar variables categóricas se utilizó la prueba de Chi cuadrado y el estadístico exacto de Fisher en caso de que ambas variables fueran dicotómicas (grado de libertad 1). Para comparar promedios de dos grupos se utilizó la prueba no paramétrica U de Mann Whitney, dado que la distribución de la muestra no fue normal incluso luego de transformarla a escala logarítmica. La significancia estadística fue del 0.05 ( $\mathrm{P}<0.05)$. 
El nivel de violencia total reportado en esta muestra fue de un $84 \%$. El tipo de violencia identificado con mayor frecuencia fue la asociada a la violencia psicológica con un 80,5\%, con al menos un episodio durante la relación de pareja. En cuanto a violencia física, un 31,2\% la experimenta, y en cuanto a la violencia sexual, un $48,8 \%$ señala haberla vivenciado (Ver tabla 1 ).

TABLA 1

Frecuencia Violencia general y Tipos Violencia

\begin{tabular}{|l|l|l|l|l|}
\hline & \multicolumn{1}{|c|}{$\begin{array}{c}\text { Violencia } \\
\text { Total }\end{array}$} & $\begin{array}{c}\text { Violencia } \\
\text { Psicológica }\end{array}$ & $\begin{array}{c}\text { Violencia } \\
\text { Física }\end{array}$ & $\begin{array}{c}\text { Violencia } \\
\text { Sexual }\end{array}$ \\
\hline No violencia & $101(16 \%)$ & $123(19,5 \%)$ & $434(68,8 \%)$ & $323(51,2 \%)$ \\
\hline Violencia & $520(84 \%)$ & $508(80,5 \%)$ & $197(31,2 \%)$ & $308(48,8 \%)$ \\
\hline \multicolumn{5}{|c}{ Nota: $\mathrm{n}=631, \mathrm{f}(\%)$}
\end{tabular}

Los ítems que se presentan con mayor frecuencia según la variable género, en el caso de las mujeres participantes son:

"Mi polola(*) me ha insultado; Mi polola desconfía y me acusa de infidelidad sin fundamento; Mi polola y yo tomamos decisiones en conjunto respecto al uso de Métodos de prevención de las Infecciones de Transmisión Sexual (ITS) incluidas SIDA-VIH entre otros; Mi polola ha tirado cosas o ha roto objetos durante una disputa o discusión; Si quiero participar en una fiesta o actividad social sin mi polola, ella está de acuerdo (ítem inverso); He perdido contacto con amistades, familiares, compañeros de carrera y/o trabajo para evitar que mi polola se moleste”. (Ver Tabla 2)

En relación a ítems contestados con mayor frecuencia en Hombres, estos fueron:

"Mi pololo (*) me ha insultado; Mi pololo desconfía y me acusa de infidelidad sin fundamento; Si quiero participar en una fiesta o actividad social sin mi pololo, él está de acuerdo (ítem inverso); Mi 
pololo ha tirado cosas o ha roto objetos durante una disputa o discusión; Mi pololo me da pellizcos y/o empujones; Mi pololo se burla de mí y me avergüenza delante de mis amigos”. (Ver Tabla 2)

TABLA 2

Ítems con mayor frecuencia según variable Género.

\begin{tabular}{|l|c|l|c|}
\hline \multicolumn{2}{|c|}{ Frecuencia Ítems Mujeres } & \multicolumn{2}{c|}{ Frecuencia Ítems Hombres } \\
\hline \multicolumn{1}{|c|}{ Ítems } & $\begin{array}{c}\text { Frecuencia } \\
\text { (f) }\end{array}$ & \multicolumn{1}{c|}{ Ítems } & $\begin{array}{l}\text { Frecuencia } \\
\text { (f) }\end{array}$ \\
\hline $\begin{array}{l}\text { Mi pololo (a) (*) me ha } \\
\text { insultado. }\end{array}$ & $213(52,1 \%)$ & $\begin{array}{l}\text { Mi pololo (a) me ha } \\
\text { insultado. }\end{array}$ & $125(56,3 \%)$ \\
\hline $\begin{array}{l}\text { Mi pololo (a) desconfía y me } \\
\text { acusa de infidelidad sin } \\
\text { fundamento. }\end{array}$ & $166(40,6 \%)$ & $\begin{array}{l}\text { Mi pololo (a) desconfía y } \\
\text { me acusa de infidelidad sin } \\
\text { fundamento. }\end{array}$ & 93 (41,9\%) \\
\hline $\begin{array}{l}\text { Mi pololo (a) y yo tomamos } \\
\text { decisiones en conjunto } \\
\text { respecto al uso de Métodos de } \\
\text { prevención de las Infecciones } \\
\text { de Transmisión Sexual (ITS) } \\
\text { incluidas SIDA-VIH entre } \\
\text { otros. }\end{array}$ & 142 (34,7\%) & $\begin{array}{l}\text { Si quiero participar en una } \\
\text { fiesta o actividad social sin } \\
\text { mi pololo (a), él / ella está } \\
\text { de acuerdo. }\end{array}$ & 74 (33,3\%) \\
\hline $\begin{array}{l}\text { Mi pololo (a) ha tirado cosas o } \\
\text { ha roto objetos durante una } \\
\text { disputa o discusión. }\end{array}$ & 137 (33,5\%) & $\begin{array}{l}\text { Mi pololo (a) ha tirado } \\
\text { cosas o ha roto objetos } \\
\text { durante una disputa o } \\
\text { discusión. }\end{array}$ & 60 (27\%) \\
\hline $\begin{array}{l}\text { Si quiero participar en una } \\
\text { fiesta o actividad social sin } \\
\text { mi pololo (a), él / ella está de } \\
\text { acuerdo. }\end{array}$ & $130(31,8 \%)$ & $\begin{array}{l}\text { Mi pololo (a) me da } \\
\text { pellizcos y/o empujones. }\end{array}$ & $\begin{array}{l}\text { Mi pololo (a) se burla de mí } \\
\text { mis amigos. }\end{array}$ \\
\hline $\begin{array}{l}\text { He perdido contacto con } \\
\text { amistades, familiares, } \\
\text { compañeros de carrera y/o } \\
\text { trabajo para evitar que mi } \\
\text { pololo(a) se moleste. }\end{array}$ & $116(28,4 \%)$ & 59 (26,6\%) \\
\hline
\end{tabular}

Nota: $\mathrm{n}:$ 631, $\mathrm{f}=(\%)$

*: Polola/pololo: chilenismo usado como sinónimo de "novio/a" o "pareja" 
En cuanto a la asociación entre tipos de violencia de pareja y variables socio demográficas, se encontró relación significativa entre género y violencia física $(\mathrm{P}=0,039)$, siendo los hombres quienes presentan mayores índices de violencia física (36,5\%) en relación a las mujeres (28,4\%) (Ver Tabla 3).

También la correlación entre violencia sexual y género muestra una relación significativa $(\mathrm{P}=0,014)$, siendo las mujeres quienes presentan mayores índices de violencia sexual (52,3\%) frente a los hombres (42,3\%) (Ver Tabla 3). Las otras correlaciones entre género y tipos de violencia no resultaron ser estadísticamente significativas.

TABLA 3

Relación entre tipos de violencia y género.

\begin{tabular}{|l|c|c|c|}
\hline & $\begin{array}{c}\text { Hombres } \\
\mathrm{N}=222\end{array}$ & $\begin{array}{c}\text { Mujeres } \\
\mathrm{N}=409\end{array}$ & $\mathrm{P}$ \\
\hline V. Psicológica & $175(79,9 \%)$ & $333(81,4 \%)$ & 0,462 \\
\hline V. Física & $81(36,5 \%)$ & $116(28,4 \%)$ & $0,039 *$ \\
\hline V. Sexual & $94(42,3 \%)$ & $214(52,3 \%)$ & $0,014^{*}$ \\
\hline
\end{tabular}

Nota: $\mathrm{n}=631, \mathrm{f}(\%),{ }^{*} \mathrm{P}<0,05$

En relación a tipos de violencia de pareja y nivel educacional, se encontró que existe una relación significativa entre violencia psicológica y nivel educacional $(\mathrm{P}=0,010)$, en que a mayor nivel educacional menor violencia psicológica (ver Tabla 4). Las otras correlaciones no fueron estadísticamente significativas. 
TABLA 4

Relación entre variables tipos de violencia y nivel educacional.

\begin{tabular}{|l|c|c|c|c|c|}
\hline & $\begin{array}{c}\text { Educación } \\
\text { Media } \\
\text { Completa } \\
\mathrm{N}=65\end{array}$ & $\begin{array}{c}\text { Superior No } \\
\text { Completa } \\
\mathrm{N}=276\end{array}$ & $\begin{array}{c}\text { Superior } \\
\text { Completa } \\
\mathrm{N}=229\end{array}$ & $\begin{array}{c}\text { Postgrado } \\
\mathrm{N}=51\end{array}$ & Valor P \\
\hline V. Psicológica & $55(84,6 \%)$ & $235(85,1 \%)$ & $\begin{array}{l}183 \\
(76,6 \%)\end{array}$ & $35(68,6)$ & $0,010^{*}$ \\
\hline V. Física & $17(8,6 \%)$ & $96(48,7 \%)$ & $69(35 \%)$ & $15(7,6 \%)$ & 0,376 \\
\hline V. Sexual & $34(11 \%)$ & $143(46,4 \%)$ & $111(36 \%)$ & $20(6,5 \%)$ & 0,292 \\
\hline
\end{tabular}

Nota: $N=631 \mathrm{f}(\%), \chi 2=11,43, * \mathrm{P}<0,05$

En relación a la variable edad, no se encontraron asociaciones estadísticamente significativas.

\section{Discusión}

Estos resultados muestran una alta prevalencia de violencia de pareja en jóvenes homosexuales en Chile, similares a los encontrados en jóvenes heterosexuales (Vizcarra y Poo, 2011; Vivanco et al., 2015), especialmente en las investigaciones realizadas en los últimos años. Esta alta prevalencia es similar a otro estudio realizado en dos países hispanoparlantes que incluyen a jóvenes homosexuales (Ortega, 2014).

Esto podría explicarse parcialmente, de acuerdo a lo señalado por diversos autores (Ortega, 2014; Moral de la Rubia y Valle, 2014; Barrientos et al., 2016) por el alto heterosexismo internalizado que se observa en jóvenes y adultos homosexuales, es decir, la interiorización de normas sociales heterocentristas discriminatorias contra los homosexuales y minorías sexuales en general, que lleva a expresar este rechazo en contra de sí mismo o en conductas violentas hacia la pareja. Sin embargo, se señala esta explicación de manera parcial, dado que el fenómeno de la violencia de pareja es un fenómeno complejo y multidimensional, lo que incluye la violencia entre parejas del 
mismo sexo, ya que si bien la literatura señala elementos comunes en este tipo de violencia entre parejas heterosexuales y homosexuales, también hay factores distintivos explicativos que se dan solo entre parejas homosexuales, como el outing forzoso y el estrés de pertenecer a una minoría como factor de vulnerabilidad en la violencia de pareja (Edwards \& Sylaska, 2013; McKenry et al., 2006; Sorenson \& Thomas, 2009; Téllez \& Walters, 2011; Villalón, 2015).

Otros factores descritos en la literatura como factores de vulnerabilidad de violencia en parejas del mismo sexo, son la invisibilización de la violencia en estas parejas, lo que pudiera generar que esta problemática perpetúe en sí la violencia, además de otros factores como el rol de género, la precariedad económica, y la doble discriminación que sufren los hombres y mujeres homosexuales, que además de la discriminación por su orientación sexual, sufren discriminación al intentar denunciar o hacer pública su situación de violencia de pareja (Téllez \& Walters, 2011; Edwards \& Sylaska, 2013; Ortega, 2014; Informe NCAVP, 2014; Brown \& Herman, 2015; Villalón, 2015).

En relación a la frecuencia de los ítems más contestados positivamente, se observa que tanto mujeres como hombres respondieron en mayor medida los mismos ítems relacionados con violencia psicológica, lo que explica el alto porcentaje de violencia psicológica encontrada en esta investigación. Esto concuerda con prácticamente todos los estudios en el tema, tanto entre parejas de jóvenes heterosexuales como homosexuales, confirmándose una vez más que la violencia psicológica es más frecuente al ser también mayormente invisibilizada y no conceptualizada como violencia, en una sociedad que legitima la violencia como método de resolución de conflictos y como forma de relacionarse (Alberdi y Matas, 2002; Vizcarra y Poo, 2011; Saldivia, 2011; Villalón, 2015).

Sin embargo, se observa en las respuestas de las mujeres, la aparición del ítem “decisiones en conjunto sobre métodos de prevención de ITS” (ítem inverso), que no está dentro de los más contestados entre los hombres, con lo 
cual se podría inferir que las mujeres lesbianas no conversan sobre este tema con su pareja, porque pueden pensar que están más protegidas de sufrir algún tipo de ITS que las mujeres heterosexuales, lo cual no es así (Sancho et al., 2012).

En los hombres, se observa una alta frecuencia del ítem "Me da pellizcos y/o empujones”, que no aparece dentro de los más contestados por las mujeres, evidenciándose la mayor prevalencia de violencia física en hombres homosexuales.

En cuanto a diferencias entre tipos de violencia encontradas de acuerdo a género, se observa en este estudio una diferencia significativa en cuanto a violencia física, siendo los hombres homosexuales quienes sufren mayor violencia física, en comparación con lesbianas. Esto es congruente con las investigaciones internacionales en el tema, que explican este fenómeno en parte por el rol de género y el machismo, que señala que los conflictos entre hombres se resuelven mediante la agresión física como parte de lo que se espera de "ser hombre”, y también en parte por el ya mencionado heterosexismo internalizado (McKenry et al., 2006; Tellez \& Walters, 2011; Murray et al., 2007; Ortega, 2014). Ortega (2014), además, refiere como hipótesis explicativa que la víctima, al auto observarse golpeando y defendiéndose físicamente, puede pensar que él también está abusando de su pareja y que no es diferente de éste, permitiendo así la aparición de la violencia física mutua en la pareja.

Otra diferencia significativa encontrada es la relación entre violencia sexual y género, la cual es sufrida en mayor medida por las mujeres homosexuales. Con respecto a este resultado no se encontró referencias similares en la literatura internacional, lo que se plantea como una interrogante y un desafío de futuras investigaciones, dado que en la literatura internacional, se encuentran pocos estudios enfocados en lesbianas específicamente, existiendo más investigaciones en relación a hombres homosexuales. No obstante, se puede vislumbrar una posible explicación de este hallazgo en este 
estudio, con el ítem que contestaron en mayor medida las mujeres “decisiones en conjunto sobre métodos de prevención de ITS”, infiriéndose, como se explicó anteriormente, que las mujeres homosexuales piensan que están más protegidas de sufrir algún tipo de ITS. Esto, como se explica en el informe elaborado por Sancho et al. (2012), es una apreciación sin fundamentos reales, dado que las mujeres lesbianas y bisexuales sufren porcentajes importantes de ITS, tanto como mujeres heterosexuales, por ejemplo, vaginosis bacteriana (VB), virus de papiloma humano (VPH), virus de inmunodeficiencia humana (VIH), entre otras infecciones, incluso aquellas que en toda su vida han tenido sexo solo con mujeres. (Sancho et al., 2012).

En relación a los tipos de violencia y nivel educacional, se encontró que a mayor nivel educacional, existe menor violencia psicológica. Estos resultados no son similares a los encontrados en otros estudios, que señalan que no existe relación entre violencia de pareja y nivel educacional (Reyes, Rodríguez y Malavé, 2005; Ortega, 2014), por lo que se hace necesario continuar indagando en la relación entre estas dos variables.

\section{LIMITACIONES DE LA INVESTIGACIÓN}

Como limitaciones de este estudio se puede mencionar que la muestra utilizada, al ser no probabilística, no asegura representatividad de la población estudiada. Esto es así ya que al ser contactados mediante redes sociales (mayoritariamente Twitter), lleva a que se mida este fenómeno en un grupo de personas con características específicas, como personas con mayor acceso a redes sociales y plataformas tecnológicas, mayor nivel educacional y posiblemente mayor nivel socioeconómico, características que no pueden generalizarse a todos los jóvenes homosexuales en Chile.

Otro aspecto que puede constituirse en una limitación, aunque también puede considerarse como fortaleza, es la rigurosidad en la categorización de 
conductas violentas, ya que la aparición de cualquiera de éstas fueron marcadas como positivas en el cuestionario pudiendo generar mayores índices de violencia (ejemplo: considerar como violencia sexual el no tomar decisiones en conjunto sobre métodos de prevención de ITS). No obstante, esta rigurosidad se debe a que se trata de un estudio pionero referente al tema en Chile y se buscó describir en detalle este fenómeno.

\section{ProYeCCIONES DE INVESTIGACIÓN E INTERVENCIÓN}

En relación a sugerencias y proyecciones de investigación, debido a que es un estudio exploratorio, se señala como primera proyección replicar este estudio en muestras más amplias, tanto cuantitativa como cualitativamente, de jóvenes homosexuales, incluyendo a quienes no acceden a redes sociales, adolescentes menores de 18 años, indagar si existen diferencias por nivel socioeconómico, representatividad de minorías étnicas, entre otras variables que pueden ser consideradas. Llama la atención que los estudios en lesbianas son escasos aún, encontrándose en la literatura mayormente investigaciones respecto a jóvenes y adultos homosexuales hombres, lo que da cuenta de que entre los propios investigadores hay un sesgo que pudiera estar influido por la sociedad patriarcal en la que estamos insertos (Fernández y Vásquez, 2013). Dada esta situación, se hace necesario ampliar y profundizar la investigación al estudio de la violencia de pareja que ocurre en lesbianas, y también a otras orientaciones sexuales, como bisexuales y transexuales, ya que en estos grupos los estudios son aún mucho más escasos.

También se considera necesario ampliar este tipo de investigaciones a adultos homosexuales, ya que en nuestro país no existen estudios al respecto, pero las investigaciones internacionales dan cuenta de que también existe una prevalencia significativa de violencia de pareja en parejas del mismo sexo adultas. 
Otros elementos que deben considerarse, son el estudio de otros factores que influyen en la violencia de pareja homosexual, como distinción entre víctima y victimario/a y factores individuales que podrían incidir en este fenómeno, por ejemplo.

Además, se sugiere la creación o adaptación en Chile de instrumentos de medición de este fenómeno que permitan una mayor pertinencia y adecuación a población de otras orientaciones sexuales, midiendo además elementos específicos descritos en la literatura internacional, que son propios de la violencia de pareja en parejas del mismo sexo, como el outing forzoso y heterocentrismo internalizado (Edwards \& Sylaska, 2013; Murray et al., 2007; Ortega, 2014).

En cuanto a sugerencias de intervención, la primera y más evidente tiene que ver con la inclusión de la población homosexual, bisexual y transexual en las políticas públicas dirigidas a detener el fenómeno de la violencia de pareja en jóvenes (y adultos) dado que, como lo muestran los resultados de esta primera investigación exploratoria, los porcentajes de incidencia son alarmantemente altos y, además, no existe ninguna institución que atienda este problema en específico en jóvenes homosexuales y de otras orientaciones sexuales.

Los profesionales de la salud y ciencias sociales, así como los policías y otros agentes encargados de prestar ayuda en estas situaciones, no se encuentran capacitados en esta temática, por lo que se sugiere comenzar a visibilizar e informar de este fenómeno, dado que, como lo señalan algunas investigaciones internacionales, cuando los jóvenes homosexuales acuden en busca de ayuda, lo que encuentran es rechazo, incomprensión y doble victimización (Sorenson y Thomas, 2009; Brown \& Herman, 2015; Informe NCAVP, 2014; Villalón, 2015). Esto ocurre porque en las sociedades aún persiste un rechazo y discriminación hacia las minorías sexuales, y esto incluye a los profesionales y agentes de ayuda, que tienden a reproducir la discriminación social, vulnerando 
y victimizando aún más a personas de estas minorías, lo que desemboca en mayores problemas de salud y sociales (Rodríguez y Vásquez, 2013; Lozano, 2014; Pérez et al., 2016). Estudios como éste, y otros que describan la invisibilización y doble victimización que viven jóvenes homosexuales, pueden contribuir a que este tema se reconozca y discuta en nuestra sociedad, ayudando a disminuir prejuicios y enfrentar esta temática desde el ámbito público y desde la sociedad civil.

Por último, cabe señalar que la visibilización de la existencia de violencia en parejas jóvenes homosexuales en Chile, permitirá a mediano o largo plazo la instauración de políticas públicas que apunten a la prevención e intervención en la violencia de pareja. Junto a esto, se sugiere crear instituciones gubernamentales o ampliar la cobertura en las instituciones ya existentes, donde las víctimas y victimario/as pudiesen acudir en solicitud de ayuda de manera segura, contribuyendo a la formación de una sociedad más justa reflejada en un Chile más igualitario.

\section{REFERENCIAS}

Agencia de Derechos Fundamentales de la Unión Europea (FRA) (2009). Informe de síntesis.

Alberdi, Inés y Matas, Natalia (2002). Violencia doméstica. Informe sobre los malos tratos a mujeres en España. Barcelona: Colección Estudios Sociales.

Amar, Mauricio (2012). El Género: conceptos, definiciones y medios de integración. Santiago de Chile: Departamento de estudios, extensión y publicaciones Biblioteca del Congreso Nacional (BCN).

Barrientos, Jaime; Gutiérrez, Karen; Ramírez, Pamela; Vega, Andrea y Zaffirri, Ivania (2016). Identidad sexual en jóvenes gay del norte de Chile. Sexualidad, salud y sociedad, 23, 118-139. Río de Janeiro: CLAMUniversidade do Estado do Rio de Janeiro.

Brown, Taylor \& Herman, Jodie (2015). Intimate Partner Violence and Sexual Abuse among LGBT People. Los Ángeles, CA: The Williams Institute UCLA. 
Cantera, Leonor (2004). Más allá del género, nuevos enfoques de nuevas dimensiones y direcciones de la violencia en la pareja. Tesis inédita de doctorado. Universidad Autónoma de Barcelona, España.

Cortés-Ayala, Lourdes; Flores, Mirta; Bringas, Carolina; Rodríguez-Franco, Luis; López-Cepero, Javier y Rodríguez, Francisco (2015). Relación de maltrato en el noviazgo de jóvenes mexicanos: análisis diferencial por sexo y nivel de estudios. Terapia Psicológica, 33, 21, pp. 5-12. Santiago: Sociedad Chilena de Psicología Clínica.

Edwards, Katie \& Sylaska, Katherina (2013). The perpetration of Intimate partner violence among LGBTQ college youth: The role of minority stress. Journal of youth Adolescence. New York: Springer Science + Business Media.

Fernández, María y Vásquez, Fernando (2013). En torno al rechazo, la salud mental y la resiliencia en un grupo de jóvenes universitarios gays, lesbianas y bisexuales. Revista Griot, 6, 1, pp. 44-65. San Juan: Universidad de Puerto Rico.

Finneran, Catherine \& Stephenson, Rob (2014) Antecedents of intimate partner violence among gay and bisexual men. Violence and victims, 29, 3, pp.422-435. United of States of America: Springer Publishing Company.

González, María Pilar (2008). Violencia en las relaciones de noviazgo entre jóvenes y adolescentes de la comunidad de Madrid. Tesis Doctoral. Madrid: Universidad Complutense de Madrid.

Hernández, Roberto, Fernández, Carlos y Baptista, Lucio (2010). Metodología de la Investigación, México: McGraw-Hill Interamericana.

Instituto Nacional de Estadísticas (INE) (2012). Resumen censo. Chile, Santiago: INE.

Instituto Nacional de la Juventud (INJUV) (2013). Séptima Encuesta Nacional de la Juventud. Santiago: INJUV.

López, Mabel y Ayala, Denisse (2011). Intimidad y las múltiples manifestaciones de la violencia doméstica entre mujeres lesbianas. Salud y sociedad, 2, pp.151-174: Universidad Católica del Norte y Universidad de Talca, Chile.

Lozano, Ignacio (2009). El significado de homosexualidad en jóvenes de la ciudad de México. Enseñanza e investigación en psicología, pp.156157. México: Consejo nacional para la investigación y la enseñanza en Psicología AC.

Lozano, Ignacio (2014). Violencia institucional homofóbica y emociones de hombres gay en la ciudad de México. Revista puertorriqueña de 
psicología, 25,2, pp.298-312. San Juan: Asociación de Psicología de Puerto Rico.

Mckenry, Patrick; Serovich, Julianne; Mason, Tina \& Mosack, Katie (2006). Perpetration of Gay and Lesbian Partner Violence: A Disempowerment Perspective. Journal of Family Violence, 21, 4, pp.233-243. New York: Springer Science + Business Media.

Moral de la Rubia, José y Valle de la O, Adrián (2014): Las dos dimensiones del rechazo hacia las personas homosexuales. Archivos de medicina, 14,1, 103-116.Manizales: Universidad de Manizales.

Movimiento de Integración y Liberación Homosexual (MOVILH) (2015). XIII Informe anual de derechos humanos de la diversidad sexual en Chile. Santiago: MOVILH. Recuperado de: http://www.movilh.cl/.

Mujika, Inmaculada y Ureta, Ana (2007). Orientación sexual: guía para jóvenes. Bilbao: Algarve.

Murray, Christine \& Mobley, A. Keith (2009). Empirical research about samesex intimate partner violence: A methodological review. Journal of Homosexuality, 56(3), pp. 361-386. USA: Taylor \& Francis Group, LLC.

Murray, Christine; Mobley, A. Keith; Buford, Anne \& Seaman-Dejohn, Megan (2007): Same-Sex Intimate Partner Violence: Dynamics, Social Context, and Counseling Implications. The Journal of LGBT Issues in Counseling, 1(4), pp. 7-30.USA: Taylor \& Francis Group, LLC.

National Coalition of Anti-Violence Programs (NCAVP) (2014). Lesbian, Gay, Bisexual, Transgender, Queer and HIV affected Intimate Partner Violence in 2013. NewYork: National Coalition of Anti-Violence Programs.

Ortega, Antonio (2014). Agresión en parejas homosexuales en España y Argentina: Prevalencias y heterosexismo. Tesis inédita de doctorado. Universidad Complutense de Madrid, Madrid.

Poo, Ana María y Vizcarra, María Beatriz (2008). Violencia de pareja en jóvenes universitarios. Terapia Psicológica, 26, 1, pp. 81-88. Santiago: Sociedad Chilena de Psicología Clínica.

Rey-Anacona, César (2008). Prevalencia, factores de riesgo y problemáticas asociadas con la violencia en el noviazgo: una revisión de la literatura. Avances en Psicología Latinoamericana, 26, 2, pp. 227-241. Bogotá: Universidad del Rosario.

Rey-Anacona, César (2013). Prevalencia y tipos de maltrato en el noviazgo en adolescentes y adultos jóvenes. Terapia psicológica, 31,2, pp. 143-154. Santiago: Sociedad Chilena de Psicología Clínica. 
Reyes, Felipe; Rodríguez, José y Malavé, Sarah (2005). Manifestaciones de la violencia doméstica en una muestra de hombres homosexuales $\mathrm{y}$ mujeres lesbianas puertorriqueñas. Revista Interamericana de Psicología, 39, 3, pp. 449-456. México DF: Sociedad Interamericana de Psicología.

Saldivia, Claudia (2011). Representaciones sociales de la violencia en el pololeo, un estudio preliminar en estudiantes universitarios. Educación y humanidades, 1, 2, pp. 83-99. Temuco: Universidad de La Frontera.

Sancho, Rubén et al. (2012). Salud sexual, VIH y otras ITS en mujeres lesbianas, bisexuales y otras mujeres que tienen sexo con mujeres (MSM). Madrid: FELGTB.

Sanhueza, Tatiana (2016). Violencia en las relaciones amorosas y violencia conyugal: convergencias y divergencias, reflexiones para un debate. Última Década, 44, pp. 133-167. Valparaíso: CIDPA.

Sorenson, Susan \& Thomas, Kristie (2009). Views of Intimate Partner Violence in Same- and Opposite-Sex Relationship. University of Pennsylvania, pp. 337-352. Pennsylvania: University of Pennsylvania.

Stephenson, Rob; Hast, Marisa; Finneran, Catherine \& Sineath, Craig (2014). Intimate partner, familial and community violence among men who have sex with men in Namibia. Culture. Health \& Sexuality, 16, 5, pp. 473-487. USA: Taylor \& Francis Group, LLC.

Tellez, Pedro \& Walters, Andrew (2011). Intimate Partner Violence Within Gay Male Couples: Dimensionalizing Partner Violence Among Cuban Gay Men. Sexuality \& Culture, pp. 153-178. New York: Springer Science + Business Media, LLC.

Tucker, Carolyn; Young, Mary; Waller, Marta; Martin, Sandra \& Kupper, Lawrence (2004). Prevalence of Partner Violence in Same-Sex Romantic and Sexual Relationships in a National Sample of Adolescents. Journal of Adolescent Health, 35, pp. 124-131.New York: Society for Adolescent Medicine.

Villalón, Lourdes (2015). La violencia en parejas homosexuales: aspectos sociales y jurídicos de la violencia en relaciones entre mujeres. Trabajo final del Máster en Criminología, Política Criminal y Sociología jurídico-penal. Barcelona: Universidad de Barcelona.

Vivanco, Ramón; Espinoza, Soraya; Romo, Cristian; Véliz, Alex y Vargas, Antonio (2015). Perpetración y victimización de la violencia en relaciones de pareja en jóvenes que cursan educación superior en la ciudad de Osorno, Chile. Polis, pp. 2-14. Santiago: CISPO. 
Vizcarra, Beatriz. y Poo, Ana María (2011). Violencia de pareja en estudiantes universitarios del sur de Chile. Universitas Psychologica, 10,1, pp. 8998. Bogotá: Editorial Pontificia Universidad Javeriana.

Recepción de artículo: diciembre 2016

Aceptación de artículo: mayo 2017 